Annales Geophysicae (2001) 19: 1107-1118 (c) European Geophysical Society 2001

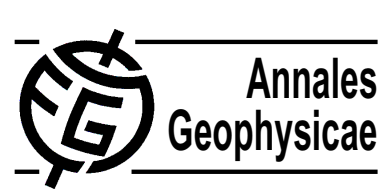

\title{
Time derivative of the horizontal geomagnetic field as an activity indicator
}

\author{
A. Viljanen ${ }^{1}$, H. Nevanlinna ${ }^{1}$, K. Pajunpääa ${ }^{1}$, and A. Pulkkinen ${ }^{1}$ \\ ${ }^{1}$ Finnish Meteorological Institute, Geophysical Research Division, P.O. Box 503, FIN-00101 Helsinki, Finland
}

Received: 20 February 2001 - Revised: 12 June 2001 - Accepted: 10 June 2001

\begin{abstract}
Geomagnetically induced currents (GICs) in technological conductor systems are a manifestation of the ground effects of space weather. Large GICs are always associated with large values of the time derivative of the geomagnetic field, and especially with its horizontal component $(d \boldsymbol{H} / d t)$. By using the IMAGE magnetometer data from northern Europe from 1982 to 2001, we show that large $d \boldsymbol{H} / d t$ 's (exceeding $1 \mathrm{nT} / \mathrm{s}$ ) primarily occur during events governed by westward ionospheric currents. However, the directional distributions of $d \boldsymbol{H} / d t$ are much more scattered than those of the simultaneous baseline subtracted horizontal variation field vector $\Delta \boldsymbol{H}$. A pronounced difference between $\Delta \boldsymbol{H}$ and $d \boldsymbol{H} / d t$ takes place at about 02-06 MLT in the auroral region when $d \boldsymbol{H} / d t$ prefers an east-west orientation, whereas $\Delta \boldsymbol{H}$ points to the south.

The occurrence of large $d \boldsymbol{H} / d t$ has two daily maxima, one around the local magnetic midnight, and another in the morning. There is a single maximum around the midnight only at the southernmost IMAGE stations. An identical feature is observed when large GICs are considered. The yearly number of large $d \boldsymbol{H} / d t$ values in the auroral region follows quite closely the $a a$ index, but a clear variation from year-to-year is observed in the directional distributions. The scattering of $d \boldsymbol{H} / d t$ distributions is smaller during descending phases of the sunspot cycle. Seasonal variations are also seen, especially in winter $d \boldsymbol{H} / d t$ is more concentrated to the northsouth direction than at other times.

The results manifest the importance of small-scale structures of ionospheric currents when GICs are considered. The distribution patterns of $d \boldsymbol{H} / d t$ cannot be explained by any simple sheet-type model of (westward) ionospheric currents, but rapidly changing north-south currents and field-aligned currents must play an important role.
\end{abstract}

Key words. Geomagnetism and paleomagnetism (geomagnetic induction; rapid time variations) - Ionosphere (ionospheric disturbances)

Correspondence to: A. Viljanen (ari.viljanen@fmi.fi)

\section{Introduction}

Geomagnetically induced currents (GIC) in power systems, pipelines and other technological conductor systems on the ground occur during rapid changes in the Earth's magnetic field (e.g. Boteler et al., 1998; Viljanen and Pirjola, 1994). However, little attention has been paid to the ionospheric current systems, which primarily produce GIC. Somewhat inexact terminology appears in literature linking GIC to electrojets, which flow approximately parallel to the (magnetic) east-west direction. In particular, this has often led to a consideration of oversimplified electrojets models, which implicitly or explicitly imply that the electric field driving GIC is primarily east-west oriented (e.g. Albertson et al., 1981; Boteler et al., 1997; Towle et al., 1992; Viljanen and Pirjola, 1994). A further inference has been that, for example, power lines parallel to geomagnetic latitudes are most prone to induction effects. A more general problem is to characterize the ionospheric current systems which can produce large GIC.

When the GIC problem is considered from the modelling viewpoint, the key quantity is the horizontal geoelectric field associated with geomagnetic variations. However, the electric field is seldom directly measured. On the other hand, GIC is closely related to the time derivative of the magnetic field $(d \boldsymbol{B} / d t)$, or more exactly, to its horizontal part (Coles et al., 1992; Mäkinen , 1993; Viljanen, 1998). Further evidence about this is given by Bolduc et al. (1998) who related the measured electric field to the time derivative of the orthogonal horizontal magnetic field. Therefore, we can base our investigation on the ground magnetic field which is available from several sites during long periods.

In an earlier work, Viljanen (1997) showed that all components of $d \boldsymbol{B} / d t$ can have large values, although the magnetic field variation is the largest in the (magnetic) northsouth direction. Coles and Boteler (1993) and Boteler et al. (1997) studied the occurrence of large time derivatives of the magnetic field components when evaluating GIC risks in Canada. We will now expand these approaches by a much 
larger data set and a more illustrative new way by studying directional distributions of the horizontal variation field vector and its time derivative. The temporal occurrence of large time derivatives will be considered from hourly to solar cycle scales.

There are several previous statistical investigations on geomagnetic variations at high latitudes based on the AU, AL and $\mathrm{AE}$ indices, with the most recent papers by Hajkowicz (1998) and Ahn et al. (2000). Our study is significantly different from theirs, because our data selection is based on the time derivative of the magnetic field, and not on the amplitude of variations. Furthermore, we concentrate on a local scale.

Among others, Olson (1986), Vennerstrøm (1999), and Ziesolleck and McDiarmid (1995) have studied ULF variations at high latitudes. Due to the equivalence of field variations and their time derivatives in the frequency domain, their results can also be interpreted in terms of $d \boldsymbol{B} / d t$. Although these authors have not based their event selections on the amplitude, there are some similarities to our results which will be discussed in this paper.

The structure of this paper is as follows: after briefly describing the data selection (Sect. 2.1), we present results from five stations in the average auroral region using a long-term data series (Sect. 2.2). A comparison between the horizontal field and its time derivative during a shorter period, but in a larger region is given in Sect. 2.3 with some remarks about seasonal variations (Sect. 2.4). The effect of the Earth's conductivity anomalies is discussed in Sect. 2.5. Finally, results based on GIC recordings are shown in Sect. 2.6.

\section{Directional distributions of the horizontal magnetic field and its time derivative}

\subsection{Selection of data}

We use data from the IMAGE magnetometer network (Fig. 1) in Fennoscandia and Svalbard (Syrjäsuo et al., 1998), and also from the preceding EISCAT magnetometer cross (Lühr et al., 1984). A reference to measured GICs will be made as well.

When studying the baseline subtracted horizontal variation field vector $\left(\Delta \boldsymbol{H}=\Delta X \boldsymbol{e}_{x}+\Delta Y \boldsymbol{e}_{y}\right)$, we select such timesteps that $|d \boldsymbol{H} / d t|>1 \mathrm{nT} / \mathrm{s}$, where the time derivative is calculated by subtracting successive values and dividing by the sampling interval (we use the simple notation $d \boldsymbol{H} / d t$, because the time derivative does not depend on baselines). In terms of GIC, $1 \mathrm{nT} / \mathrm{s}$ typically causes a current of a few amperes in the Finnish high-voltage power system or the natural gas pipeline.

An approximate rule for interpreting vector distributions is that $\Delta \boldsymbol{H}$ can be converted to an overhead equivalent ionospheric current by a $90^{\circ}$ clockwise rotation. When $d \boldsymbol{H} / d t$ is rotated $90^{\circ}$ anticlockwise, it is parallel to the horizontal electric field at the Earth's surface (strictly true in the fre-

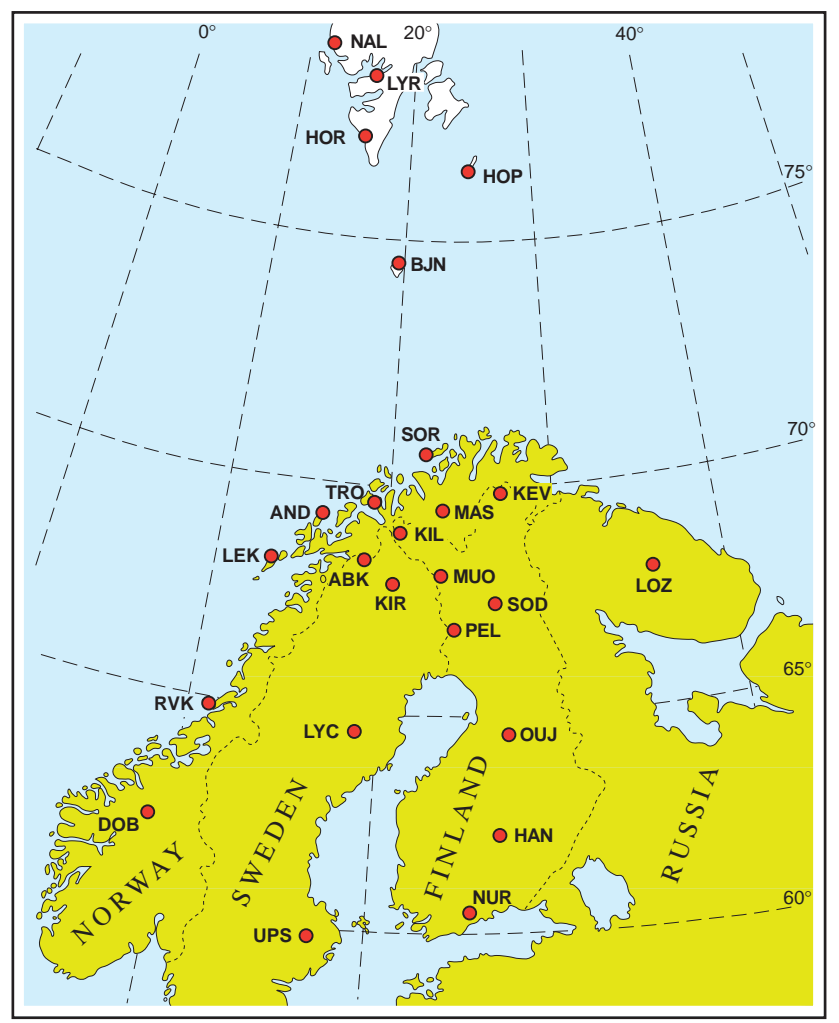

Fig. 1. IMAGE magnetometer stations in 2000. Geographic latitudes and longitudes are shown in the figure. Geomagnetic latitudes are approximately obtained by subtracting $3^{\circ}$ from the geographic ones.

quency domain if the disturbance field is a plane wave and the Earth's conductivity depends only on depth).

In the following, we primarily ignore stations in Svalbard, because they are located at latitudes farther north of all ground technological systems which presently can experience $\mathrm{GIC}$.

\subsection{Distributions of $d \boldsymbol{H} / d t$ in the auroral region}

The best temporal data coverage is provided by five IMAGE stations in the average auroral region (PEL, MUO, KIL, $\mathrm{KEV}$, SOR), of which there are simultaneous and nearly continuous data available since 1982-1983. Directional distributions of $d \boldsymbol{H} / d t$ are shown in Fig. 2. For better readability, the distribution patterns are normalized separately for each subplot. The total number of vectors used is given under the subplots. The most striking features are the narrow distributions especially from 1984 to 1987 and from 1994 to 1996 , when the (geomagnetic) north-south direction is preferred. On the other hand, distributions are much more uniformly scattered between 1988 to 1992 and 1997 to 2000. The explanation for these discrepancies may be related to different solar activity conditions. During descending parts of sunspot cycles (1984-1987, 1994-1996), magnetic storms are primarily caused by 27 days of recurrent activity, and during ascending parts (1988-1992, 1997-2000), they are dominantly 

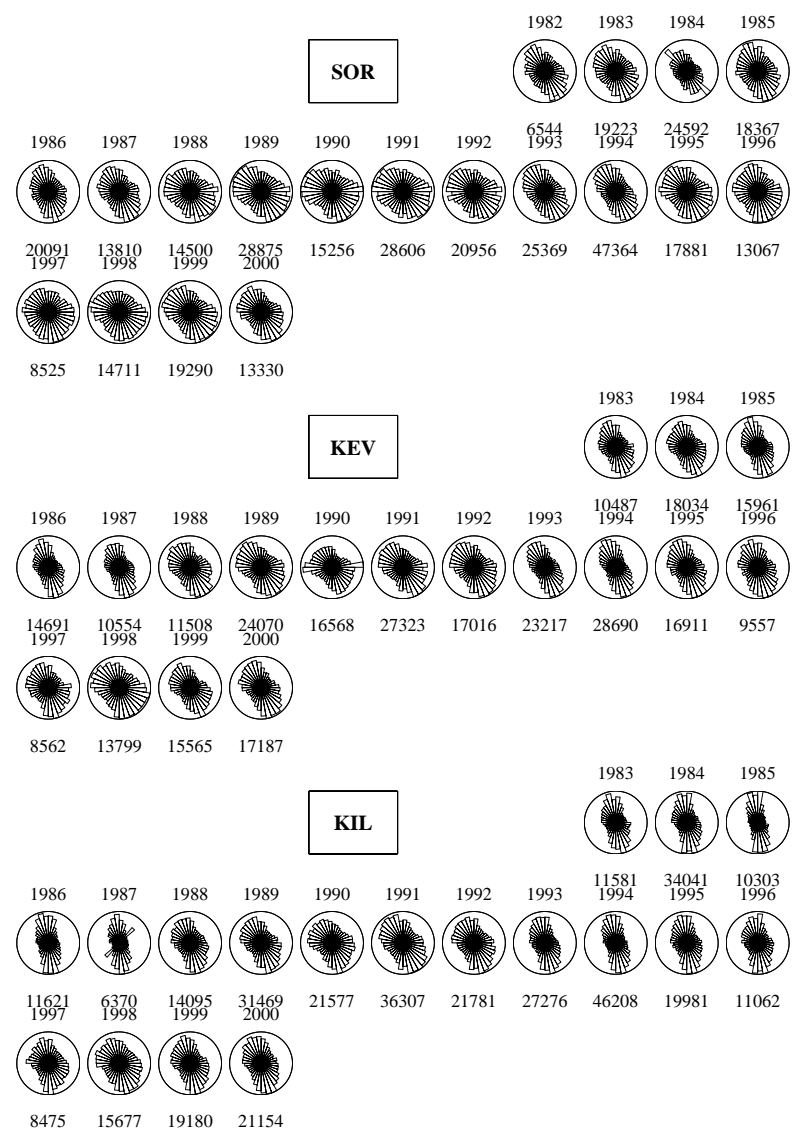

$\begin{array}{lllll}1982 & 1983 & 1984 & 1985\end{array}$



Fig. 2. Directional distributions of $d \boldsymbol{H} / d t$ at Sørøya (SOR), Kevo (KEV), Kilpisjärvi (KIL), Muonio (MUO) and Pello (PEL) from 1982 to 2000. Only timesteps fulfilling the condition $|d \boldsymbol{H} / d t|>$ $1 \mathrm{nT} / \mathrm{s}$ are included. The sampling interval was $20 \mathrm{~s}$ until November 1992 , and after that $10 \mathrm{~s}$. For consistency, $10 \mathrm{~s}$ values were averaged to $20 \mathrm{~s}$. The eleven years of the sunspot cycle 22 are in the middle row. The geographic north direction is upwards. The distribution patterns are normalized separately for each year for better readability. The number of timesteps is given under each subplot. Data from 1982 to 1983 were available only of short periods, and only about half of 1985 to 1987 was covered at KIL. The geomagnetic latitudes of the stations are between $63^{\circ}$ and $67^{\circ}$.


Fig. 3. Relative number of datapoints used in Fig. 2. Upper panel: yearly average of all five stations; the dotted line is the yearly average of the $a a$ index (obtained from the National Geophysical Data Center). Lower panel: average value at stations from 1982 to 2000. The sampling interval used here is $20 \mathrm{~s}$, which was the original one for data from 1982 to 1992 . The newer $10 \mathrm{~s}$ data were averaged to $20 \mathrm{~s}$ values too.

transient. Transient storms are usually more intense, but how this affects $d \boldsymbol{H} / d t$ distributions is unclear.

The relative number of datapoints with $|d \boldsymbol{H} / d t|>1 \mathrm{nT} / \mathrm{s}$ compared to the $a a$ index is shown in Fig. 3. The relative number of points at each station (lower panel) is larger at KIL than at KEV, although they are approximately at the same magnetic latitude. The possibility that this is caused by the Earth's conductivity will be discussed in Sect. 2.5. The yearly occurrence correlates well with the $a a$ index. Some periodicity is seen, with the smallest number (1987, 1997) occurring a little after the minimum of the sunspot cycle $(1986,1996)$. However, it is peculiar that in both 1987 and 1994 one can see narrow distributions in Fig. 2, although their $d \boldsymbol{H} / d t$ activity levels are the smallest and highest ones in Fig. 3. The least and most scattered years $(1987,1998)$ are separated by 11 years, i.e. one solar cycle.

Vennerstrøm (1999) considered the power spectral density of 2-4 min, and the 5-10 min frequency bands of the Greenland magnetometer chain during the morning hours (0610 MLT). She found a good correlation between the power spectral density and $a a$ at the southern stations of the chain, located at about the same magnetic latitudes as the five stations in Fig. 2. This result is clearly equivalent to that shown in the upper panel of Fig. 3 since large $d \boldsymbol{H} / d t$ values are associated with short-period variations. The similarity is interesting since we have considered all local times, and Vennerstrøm (1999) considered only the local morning, which primarily lacks, for example, substorm activity. However, as will be seen in Sect. 2.3, the local morning hours have a significant contribution to the total number of large $d \boldsymbol{H} / d t$ at auroral latitudes.

The newest studies showing the great variability between years and solar cycles in terms of the global AL, AU and AE 
indices are presented by Hajkowicz (1998) and Ahn et al. (2000). However, any evident correlation to the variability of $d \boldsymbol{H} / d t$ is hard to find due to the very different data selection criteria compared to our study.

According to these results, a considerable variation of the $d \boldsymbol{H} / d t$ distributions from year-to-year, and from site-to-site, is a clear fact. Next, it is interesting to study the simultaneous distributions of the horizontal variation field $\Delta \boldsymbol{H}$.

\subsection{Distribution of $\Delta \boldsymbol{H}$ and $d \boldsymbol{H} / d t$ at all IMAGE stations}

Our basic dataset for the $\Delta \boldsymbol{H}$ investigation consists of all available values from 1994 to 2000 at the IMAGE magnetometer stations (with the condition $|d \boldsymbol{H} / d t|>1 \mathrm{nT} / \mathrm{s}$ ). Since we currently study field variations, quiet time baselines are needed. They have been selected visually and separately for each day. Although this is somewhat subjective, for the present purpose, small uncertainties are unimportant (time derivatives do not depend on baselines). For computational convenience, we now consider one minute values.

An example of distributions at Kilpisjärvi (KIL) located in the average auroral zone, and Oulujärvi (OUJ) in the subauroral region is shown in Fig. 4, where the distribution patterns are normalized separately for each subplot. The main direction of $\Delta \boldsymbol{H}$ is to SSE, which is equivalent to an overhead WSW directed ionospheric current parallel to the local geomagnetic latitude. The eastward electrojet has a relatively significant contribution only during some years and only at the southernmost sites. The directional distribution of $\Delta \boldsymbol{H}$ is nearly identical from year-to-year, whereas the distribution of $d \boldsymbol{H} / d t$ clearly varies.

The number of included timesteps with $20 \mathrm{~s}$ values is 5 to 6 times that of one minute values (Figs. 2 and 4). In addition to the larger number of $20 \mathrm{~s}$ datapoints, one-minute averaging smooths out many spikes. However, yearly distributions of $d \boldsymbol{H} / d t$ do not change much. Concerning $\Delta \boldsymbol{H}$ distributions, it does not matter whether $10 \mathrm{~s}$ or $20 \mathrm{~s}$ or one minute data are used.

Most timesteps of large $|d \boldsymbol{H} / d t|$ are associated with active periods, as shown by the distributions of $|\Delta \boldsymbol{H}|$ depicted in Fig. 5. When all available one-minute values from 1994 to 2000 are used, the average $|\Delta \boldsymbol{H}|$ is $33 \mathrm{nT}$ at OUJ, and $60 \mathrm{nT}$ at KIL, which are much smaller than the ones in Fig. 5. The difference in the shape of the distributions between KIL and OUJ indicates that large $|d \boldsymbol{H} / d t|$ values at subauroral latitudes are more regularly associated with exceptionally large geomagnetic variations. This is the case at all subauroral IMAGE stations.

Directional distributions of $\Delta \boldsymbol{H}$ and $d \boldsymbol{H} / d t$ at the continental IMAGE stations are presented in Fig. 6. This example concerns the year 2000 when there were 25 stations operating, and the solar cycle maximum was reached. The common feature of $\Delta \boldsymbol{H}$ at all sites is its narrow orientation along the local magnetic meridian to SSE. The time derivative is more varying, although there is a clear concentration along the NNW-SSE line at subauroral sites. The tilted $d \boldsymbol{H} / d t$ pattern due to a conductivity anomaly at LYC will be dis-

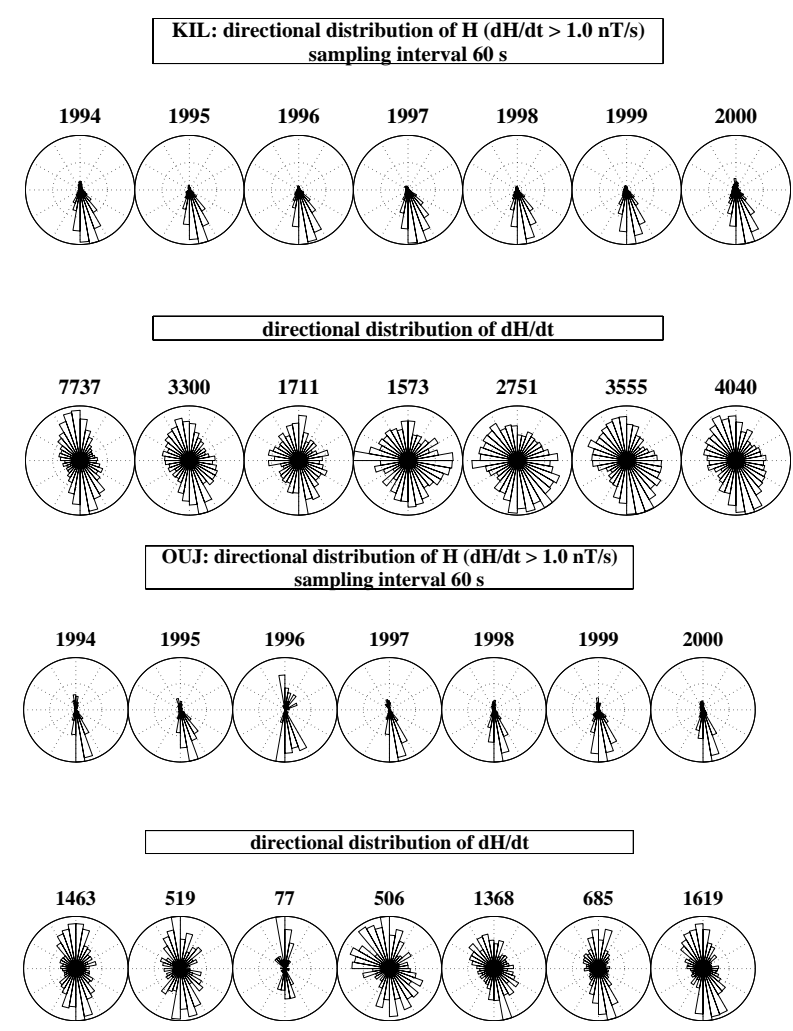

Fig. 4. Directional distribution of $\Delta \boldsymbol{H}$ (upper row) and $d \boldsymbol{H} / d t$ (lower row) at Kilpisjärvi (KIL) and Oulujärvi (OUJ) from 1994 to 2000 , as calculated from one minute values. Only timesteps fulfilling the condition $|d \boldsymbol{H} / d t|>1 \mathrm{nT} / \mathrm{s}$ are included. The distribution patterns are normalized separately for each subplot. The total number of timesteps are given as title texts in the lower row.

cussed in Sect. 2.5. Data from LOZ in 2000 is not shown due to an instrumental problem which biased the statistical results. Inspection of earlier years showed that LOZ resembles SOD and PEL, which are approximately located at the same geomagnetic latitude.

For clarity, the vector distributions are scaled separately for each site in Fig. 6. The relative number of large values is illustrated in Fig. 7. It is smallest at southern sites and then increases northward. The relative numbers at the Svalbard stations (not shown in the figure) are $0.75 \%(\mathrm{BJN}), 0.80 \%$ (HOP), $0.66 \%$ (HOR), $0.39 \%$ (LYR), and $0.22 \%$ (NAL), so the $d \boldsymbol{H} / d t$ activity decreases northward of the average auroral region. This behaviour is typical for all local times, as also shown by Boteler et al. (1997) when considering the occurrence of hourly maxima of $d X / d t$ over $1 \mathrm{nT} / \mathrm{s}$ in Canada.

We also calculated the daily sum of large $|d \boldsymbol{H} / d t|$ values at all IMAGE stations and compared it to the $A_{k}$ index of Nurmijärvi. The correlation coefficient between these two activity indicators varies between $0.71-0.87$ from 1994 to 2000. Consequently, the daily activity can be described in terms of $|d \boldsymbol{H} / d t|$ as well.

The hourly distribution of large $|d \boldsymbol{H} / d t|$ values at a subauroral (NUR), auroral (KIL) and cusp/polar cap station (LYR) 

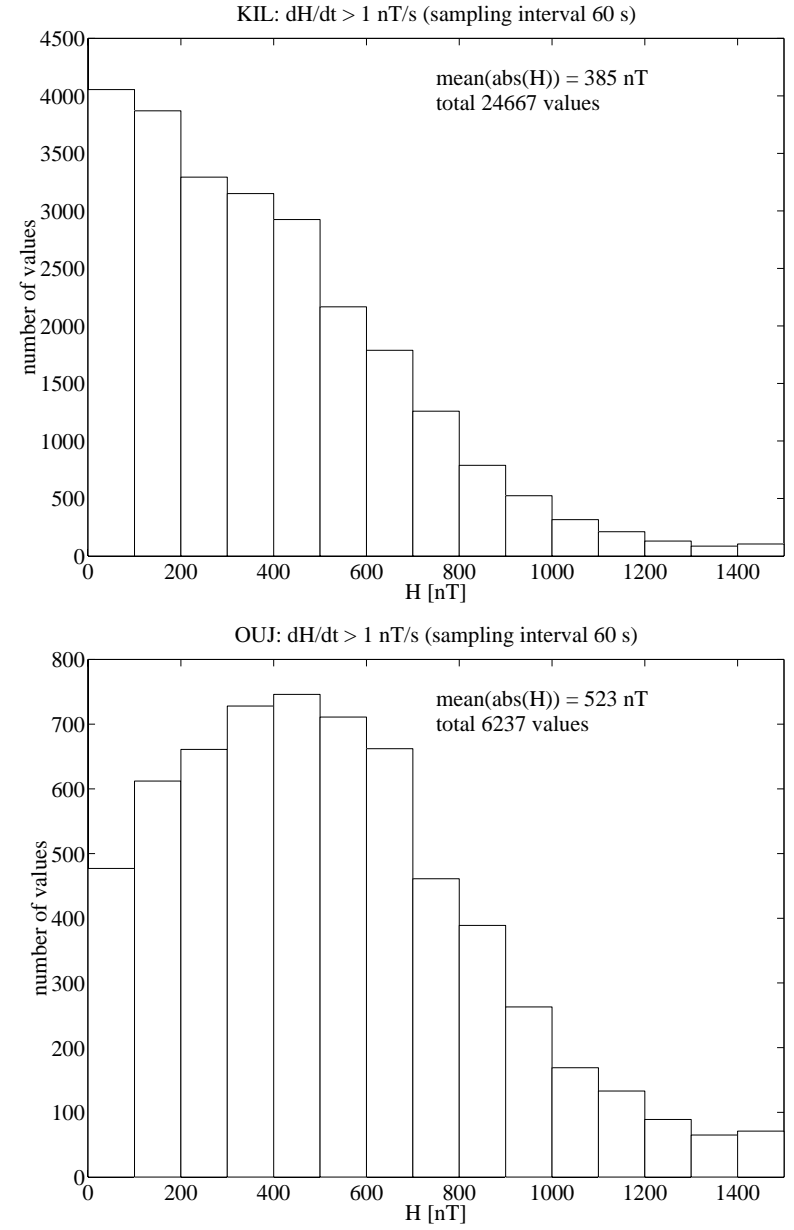

Fig. 5. Distribution of the amplitudes of the $\Delta \boldsymbol{H}$ vectors used in Fig. 4. When all available values from 1994 to 2000 are used, the averages are $33 \mathrm{nT}$ at $\mathrm{OUJ}$ and $60 \mathrm{nT}$ at KIL.

in 2000 is presented in Fig. 8. The magnetic local time (MLT) used here is the one associated with the solar magnetic coordinate system. It is obtained from the GSM system by a rotation around the $y$ axis so that $z$ is parallel to the dipole axis. The difference between UT and MLT was simply taken as an average of the whole year for each station separately. For statistical purposes this is sufficiently accurate (the solar MLT is 10-20 minutes ahead of the CGM MLT in the mainland stations). Only the year 2000 is shown here, but we also calculated the corresponding results from 1994 to 1999 , which basically have a similar behaviour.

The diurnal behaviour at the auroral region (KIL) is consistent with the directional distribution of $\Delta \boldsymbol{H}$, in which the nighttime westward electrojet dominates. The midnight maximum can be related to the substorm activity, and the morning peak can be related at least partly to pulsations and omega bands. Typical pulsations such as Pc5's with periods of 150-600 s and amplitudes of $100 \mathrm{nT}$, can produce $|d \boldsymbol{H} / d t|$ up to $2 \pi 100 / 150 \mathrm{nT} / \mathrm{s} \approx 4 \mathrm{nT} / \mathrm{s}$.

The double peak in Fig. 8 is most pronounced at stations in the average auroral region, which is in accordance with the
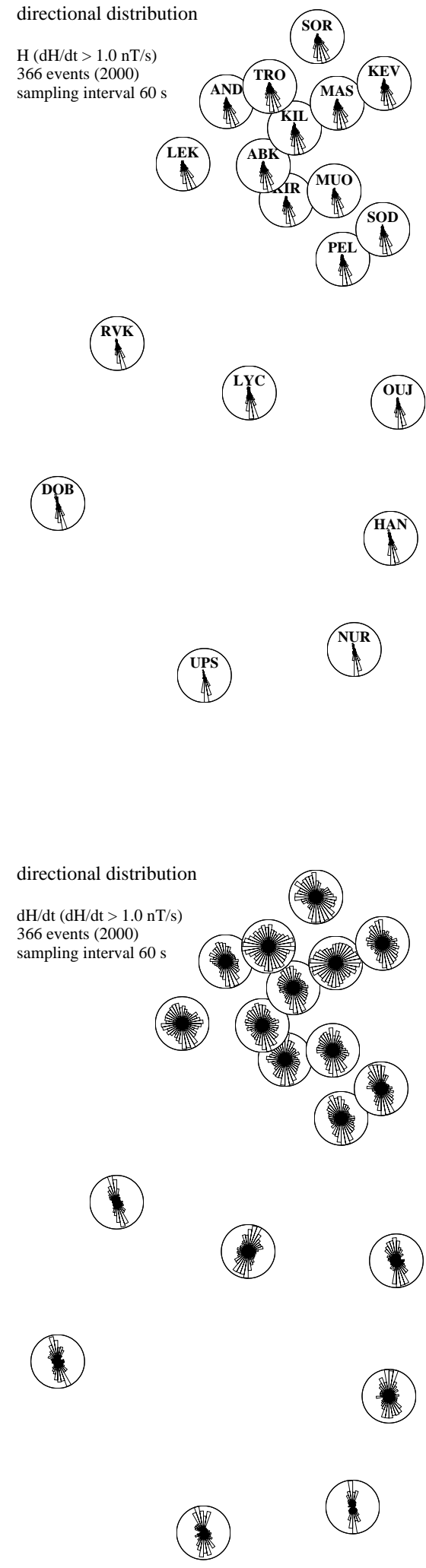

Fig. 6. Directional distributions of $\Delta \boldsymbol{H}$ and $d \boldsymbol{H} / d t$ at the continental IMAGE stations in 2000 (from one-minute values with the condition $|d \boldsymbol{H} / d t|>1 \mathrm{nT} / \mathrm{s})$. 


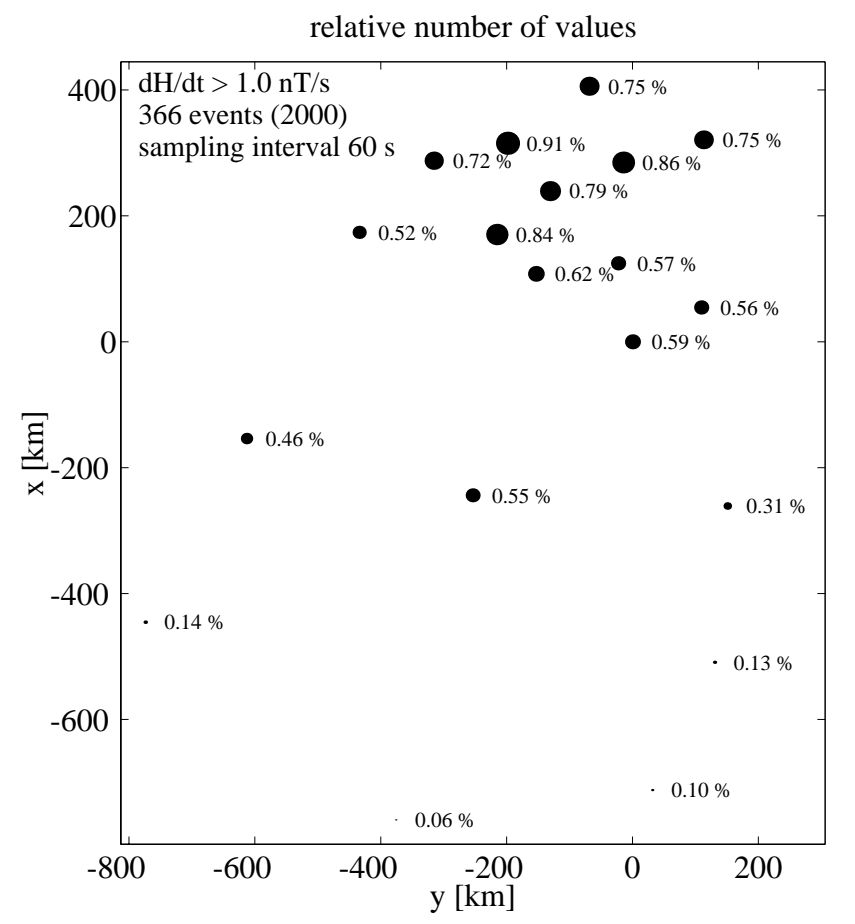

Fig. 7. Relative number of data points fulfilling the condition $|d \boldsymbol{H} / d t|>1 \mathrm{nT} / \mathrm{s}$ at each station in Fig. 6. The radius of each circle is proportional to the percentage.

results of Coles and Boteler (1993), Boteler et al. (1997) (replotted in Boteler et al., 1998). ULF studies also reveal the same feature (e.g. Vennerstrøm, 1999; Ziesolleck and McDiarmid, 1995); the years 1996 and 1997 even have a slight triple structure. At the southernmost sites (UPS, NUR), the early morning minimum is sometimes missing (e.g. 2000), but at times, it is observable (e.g. 1998). The double peak was also found by Viljanen (1997) in terms of the average value of $d \boldsymbol{B} / d t$, but he did not discuss the subject further.

At the Svalbard stations the morning maximum typically occurs around 10 MLT (latest in the north, as found by Boteler et al., 1997, as well) and the evening maximum just before the local midnight, as at more southern latitudes, but the variability from year-to-year is large. Furthermore, there are often three clearly distinct maxima, as at LYR in 2000. Olson (1986) studied Pc3-5 pulsations at Cape Perry (magnetic latitude about $74^{\circ}$ ) and found the occurrence maxima at about 9 MLT and 19 MLT, i.e. somewhat earlier than at LYR (magnetic latitude 75).

Although ULF studies show that variations in certain frequency bands have the same daily occurrence probability, some care will be needed in the interpretation. As mentioned by Vennerstrøm (1999), it is not possible to distinguish the ULF band power due to different physical phenomena, such as broadband noise, $\mathrm{Pi}$ and Pc pulsations, impulse events and substorm expansions. Therefore, in future studies, quantitative models of ionospheric currents during large GIC events are needed.

Nevanlinna and Pulkkinen (2001) have studied the diur-
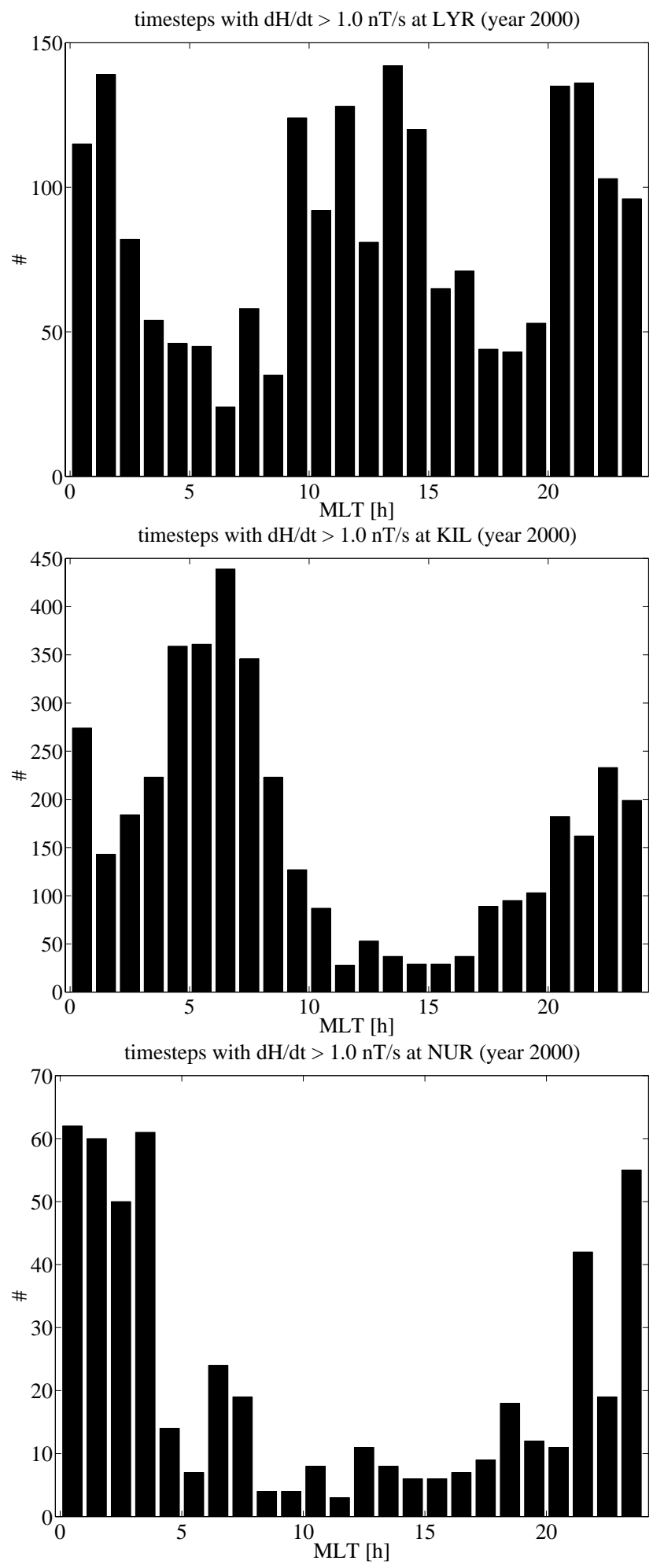

Fig. 8. Diurnal variation of the number of timesteps in 2000 when $|d \boldsymbol{H} / d t|$ exceeded $1 \mathrm{nT} / \mathrm{s}$ at three IMAGE magnetometer stations (one-minute data). LYR is located in the cusp/polar cap, KIL in the auroral region, and NUR in the subauroral region. The magnetic local time (MLT) is given in the solar magnetic coordinate system (roughly, MLT $=\mathrm{UT}+3 \mathrm{~h}$ ). 
nal occurrence of auroras in north Finland using all-sky camera data from 1973 to 1997 . The mean hourly occurrence of auroras has nearly a Gaussian distribution centered at the local magnetic midnight. The probability of seeing auroras is slightly higher in the morning hours, but a double peak, such as the one at the auroral region station KIL in Fig. 8, does not exist. There is a double peak, although it is not very pronounced, only when the auroral occurrence is considered during rare severe storms. In addition, these maxima are at about 16 and 04 MLT, which differ from those of $|d \boldsymbol{H} / d t|$.

We also calculated the hourly distribution for a few stations, of which SOR is shown as an example in Fig. 9. Each hourly pattern is again normalized separately, and the relative number of datapoints is given under each subplot. A very similar behaviour was found at MUO as well. Although the selection of data is based on the large $|d \boldsymbol{H} / d t|$ condition, the $\boldsymbol{H}$ distribution reflects the familiar diurnal pattern of ionospheric currents. The daytime convection (8-16 MLT) with the turning of the electrojet from the westward to the eastward direction is clear (11-13 MLT). The Harang discontinuity at 18-20 MLT is also distinctly manifested with the rotation of the $\Delta \boldsymbol{H}$ pattern from the north to the south through the west. The rest of the time is governed by westward currents.

The $d \boldsymbol{H} / d t$ vector follows in many respects what one could expect: in the daytime and in the evening, before midnight, it is primarily parallel (or antiparallel) to $\Delta \boldsymbol{H}$. However, after midnight, until the morning, around the noon convection reversal, and around the Harang discontinuity, the time derivative is much more scattered. Its most peculiar feature is a strong concentration at 03-05 MLT parallel to the east-west line, perpendicular to the main ionospheric current. At times a kind of asymmetry is also seen, for example, at 20-24 MLT, when substorms typically occur. Large $d \boldsymbol{H} / d t$ vectors tend to point more frequently to the south than to the north. A plausible explanation is that the southward $d \boldsymbol{H} / d t$ 's are associated with auroral breakups with rapidly decreasing $\Delta X$. The increase of $\Delta X$ back to quiet time levels is slower, causing smaller time derivatives not counted in statistics.

At lower latitudes (e.g. OUJ), the $d \boldsymbol{H} / d t$ pattern is primarily north-south oriented throughout the day (Fig. 6). In particular, there is no east-west turning in the early morning as at higher latitudes.

To investigate the morning hours in more detail, we made a simple test by considering only UT hours 0-5 (MLT 3-8) of the year 2000. The EW orientation of $d \boldsymbol{H} / d t$ is dominating at auroral latitudes, but at southern sites, the vector pattern is nearly identical to the one in Fig. 6. The 5 most active days yield $32.3 \%$ of all large $|d \boldsymbol{H} / d t|$ values (23 January, 17 and 24 May, 12 August, 13 October). A visual inspection of these days showed that the large morning hour events seem to be a mixture of a substorm and pulsation activity. We repeated this test for 1994, which has a narrow $d \boldsymbol{H} / d t$ pattern at auroral latitudes (Fig. 2). Now there was no tendency to the EW direction. However, the 5 most active days (yielding $21.9 \%$ of all values) again look qualitatively similar to the ones mentioned above in the year 2000. The narrowness of

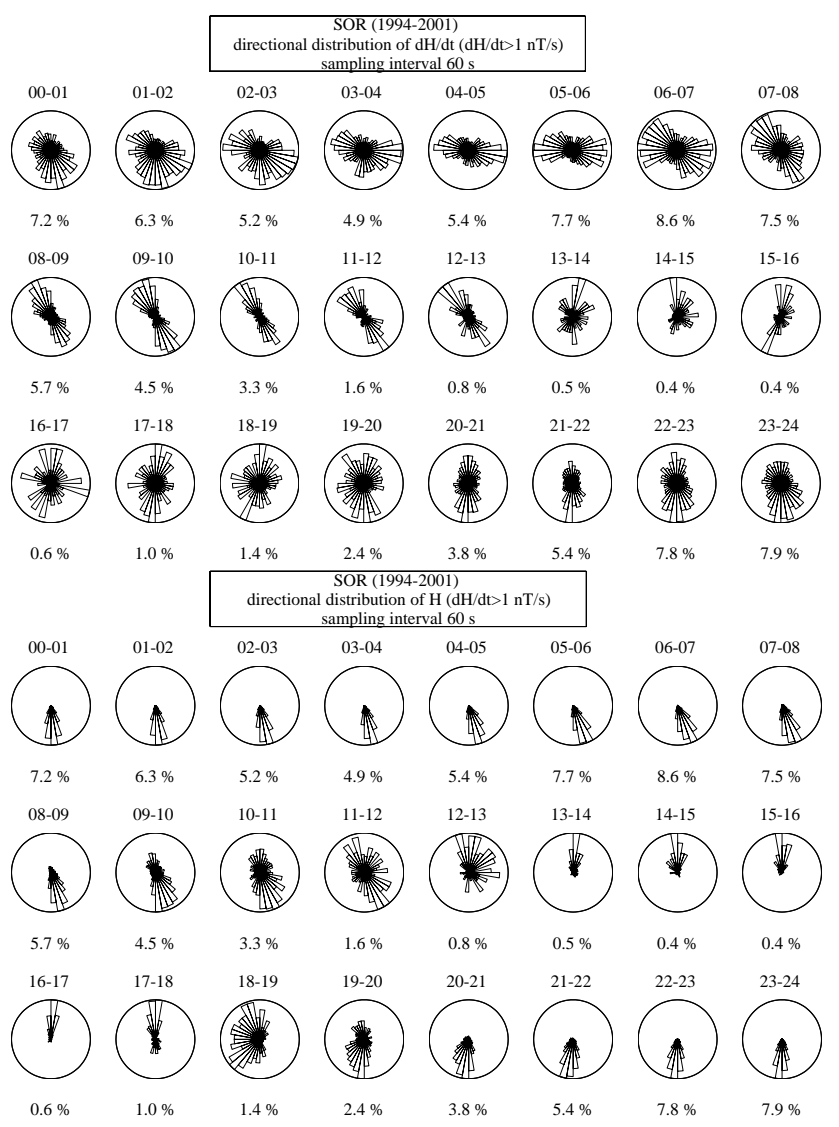

Fig. 9. Hourly distribution patterns of $d \boldsymbol{H} / d t$ and $\Delta \boldsymbol{H}$ at SOR from 1994 to 2001 for timesteps when $|d \boldsymbol{H} / d t|$ exceeded $1 \mathrm{nT} / \mathrm{s}$ (one-minute data). The percentage of each hour of the whole day is given under subplots. Title texts show the magnetic local time (MLT) in the solar magnetic coordinate system (MLT $=\mathrm{UT}+3 \mathrm{~h}$ 16 min for all timesteps used here).

the overall 1994 patterns in Fig. 2 is due to the fact that the contribution of the morning hour events in 1994 to the total distribution is only about half of that in 2000.

Although the aim of this paper is not to model ionospheric currents causing large $d \boldsymbol{H} / d t$, some purely mathematical considerations are easily performed. We limit this exercise to horizontal ionospheric currents. Assuming of a layered conductivity structure of the Earth, field-aligned currents can be replaced with horizontal currents, thus producing the same total magnetic field and the same horizontal electric field at the Earth's surface as the true current system (Pirjola and Viljanen, 1998).

In thinking about the $\Delta \boldsymbol{H}$ and $d \boldsymbol{H} / d t$ patterns at 2024 MLT in Fig. 9, a simple westward sheet current model can explain these patterns. It produces a southward horizontal variation field, and amplitude variations make the time derivative to point to the north or south. To model the pattern at 03-06 MLT, we can still assume a westward sheet current. To produce an east-west oriented $d \boldsymbol{H} / d t$, a very small rotation of the sheet current is sufficient. Assume that the length of $\Delta \boldsymbol{H}$ does not vary (e.g. $300 \mathrm{nT}$, Fig. 5), but that its 
direction changes $2^{\circ}$ in $10 \mathrm{~s}$. Then $d \boldsymbol{H} / d t$ is practically eastward, and its magnitude is $1 \mathrm{nT} / \mathrm{s}$. However, we could as well imagine that the westward current does not change at all ( $X$ constant, for example, $300 \mathrm{nT}$ ), but that there is a northward current whose amplitude changes rapidly, for example, causing $Y$ to increase from zero to $10 \mathrm{nT}$ in $10 \mathrm{~s}$. Consequently, $|d \boldsymbol{H} / d t|=1 \mathrm{nT} / \mathrm{s}$, but $\boldsymbol{\Delta} \boldsymbol{H}$ is practically unaffected.

Inspection of Fig. 6 shows that a simple sheet current model explains the behaviour of $\Delta \boldsymbol{H}$, but leads to conflicts with $d \boldsymbol{H} / d t$. Namely, a sheet current should produce an identical pattern of $d \boldsymbol{H} / d t$ everywhere, which is not the case. A logical solution is that small-scale structures with currents in all directions appear regularly in the auroral region (e.g. MUO, MAS, SOR), but their effects are not seen far away in the south (e.g. NUR, HAN, OUJ). Therefore, subauroral sites are more affected by changes in largescale current systems. Details remain to be investigated further, since large magnetic disturbances at subauroral latitudes seem to differ from those in the average auroral zone. Finally, there are large collections of well-established models based on different observations of the ionosphere (e.g. Untiedt, 1993; Amm, 1995). These models do have currents in all directions. When the models are applied in GIC calculations, $d \boldsymbol{H} / d t$ and the accompanied ground electric field are seen to have large values in all directions (Viljanen et al., 1999).

The limit of $1 \mathrm{nT} / \mathrm{s}$ used throughout this paper guarantees that only large GIC events are included. We also made a test by using a limit of $2 \mathrm{nT} / \mathrm{s}$, and replotted Figs. 6 and 8 . The relative number of values decreased approximately by a factor of 5, but no essential changes in the directional patterns nor in the diurnal behaviour were found. To study the most extreme events, we took all IMAGE data from 1994 to 2000 and considered $d \boldsymbol{H} / d t$ 's larger than $20 \mathrm{nT} / \mathrm{s}(10 \mathrm{~s}$ sampling interval). 243 vectors in total were found, and half of them were from the Svalbard stations. The $\boldsymbol{H}$ vectors were directed to SSE, and the derivative vectors uniformly to all directions. When only the mainland stations were considered, the EW direction of $d \boldsymbol{H} / d t$ was slightly prefered. Consequently, small-scale ionospheric phenomena seem to be important during the largest storms as well.

\subsection{Seasonal variations}

To study seasonal changes, we considered the same five IMAGE stations as in Sect. 2.2 and used the data of the sunspot cycle from 1986 to 1996 . We divided each year into seasons as follows: winter (1 January to 4 February, 6 Novomber to 31 December), spring (5 February to 6 May), summer ( 7 May to 5 August), and autumn (6 August to 5 November). The corresponding $d \boldsymbol{H} / d t$ distributions are shown in Fig. 10 . Winter with its narrow $d \boldsymbol{H} / d t$ patterns is clearly different from the other seasons. Summer has the most scattered distributions, but the difference between spring and autumn is not pronounced. Quite a clear seasonal effect is observed in the relative number of large $|d \boldsymbol{H} / d t|$ as an average of the five sites: winter $0.9 \%$, spring $2.0 \%$, summer $0.9 \%$ and autumn
$1.5 \%$ (20 s sampling interval). This is in agreement with the general increase of geomagnetic activity near equinoxes (e.g. Russell and McPherron, 1973). Coles and Boteler (1993) state that only the June minimum in the $d \boldsymbol{B} / d t$ activity is clear, but they only calculated the number of hours when at least one component of $d \boldsymbol{B} / d t$ exceeded $5 \mathrm{nT} / \mathrm{s}$ in oneminute data. On the other hand, the reproduction of historical GIC observations by Boteler et al. (1998) shows that the sum of telegraph disturbances in Norway from 1881 to 1884 had very clear peaks at equinoxes. Therefore, the lack of a clear seasonal dependence in Coles and Boteler (1993) may be due to the data binning.

\subsection{Effect of the Earth's conductivity}

Most of the continental IMAGE stations are located on the Fennoscandian shield, but those in the north and west close to the Atlantic Ocean lie on the Caledonides. The area is known to have many good conducting zones in the crust (Korja and Hjelt, 1993; Pajunpää, 1987). Although only Lycksele (LYC) is known to be directly above one major conductivity anomaly, there are a few other stations where the directional distribution of $d \boldsymbol{H} / d t$ is clearly affected by telluric currents.

LYC is located on the southwestern edge of a large electrical conductivity anomaly in the crust. This Skellefte anomaly, modelled by Rasmussen et al. (1987), had a very low resistivity ( $4 \Omega \mathrm{m}$ ) in the upper and middle crust (down to about $20 \mathrm{~km}$ near LYC). The main WNW strike of the anomaly is in a good agreement with the anomalous $d \boldsymbol{H} / d t$ distribution at LYC in Fig. 6. The induced currents enhance the time derivative of the magnetic field perpendicular to the strike of the anomaly. This is seen as a relatively large occurrence probability at LYC in Fig. 7. The reason why the $\Delta \boldsymbol{H}$ distribution is not affected by the anomaly is that in the frequency spectrum of $|\Delta \boldsymbol{H}|$, long periods dominate. On the contrary, the spectrum $|d \boldsymbol{H} / d t|$ is peaked at shorter periods. This corresponds to smaller skin depths in the Earth, so the telluric currents at short periods are concentrated in the anomaly.

MAS has a remarkably different directional distribution when compared to the neighboring sites KIL and KEV. The reversed real induction arrow at MAS, calculated by Viljanen et al. (1995), points towards WSW and has its maximum length at a few hundred seconds. The amplitude of the variations in the east-west direction is also larger when compared to KIL and KEV. TRO has a similar directional distribution of $d \boldsymbol{H} / d t$, as MAS, but we have no a priori information about the conductivity structure there. At the nearby AND, the distribution is quite narrow, which is believed to be caused by a strong conductivity anomaly north of the station.

The larger relative number of $|d \boldsymbol{H} / d t|$ values greater than $1 \mathrm{nT} / \mathrm{s}$ at KIL compared to KEV (Fig. 3) may be related to a better conductivity of the uppermost Earth layers at KIL, located in the northern part of Caledonides. However, this is only speculative, since we do not have definite information about the local conditions. 

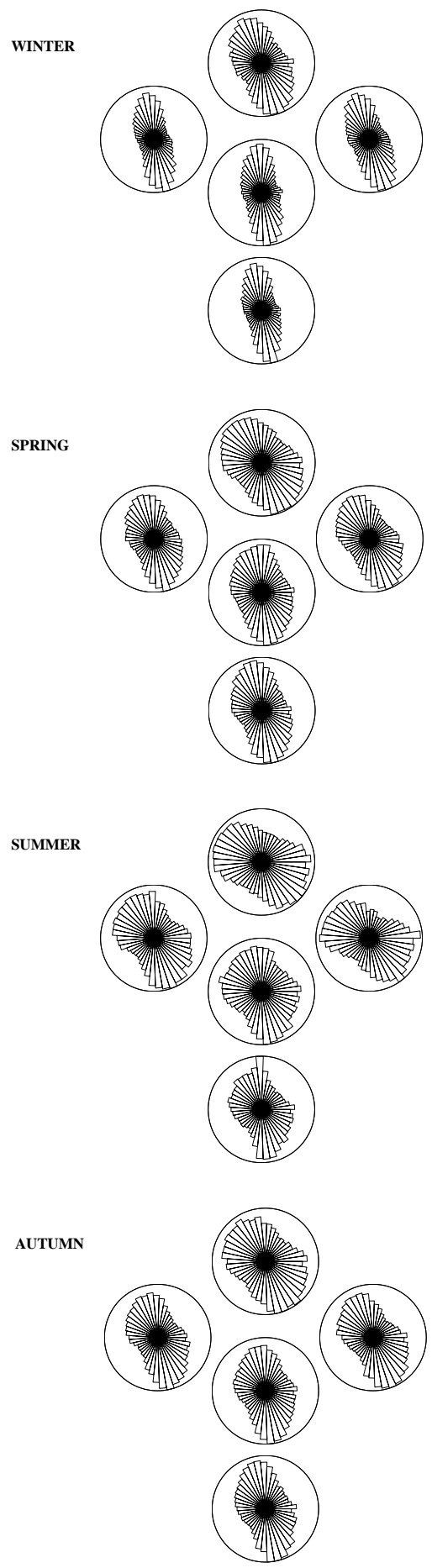

Fig. 10. Directional distributions of $d H / d t$ during the four seasons from 1986 to 1996 at PEL, MUO, KIL, KEV, SOR. Panels from top: winter, spring, summer, autumn. Stations in the middle are SOR (top), MUO, PEL (bottom). KIL is on the left and KEV on the right. The distribution patterns are normalized separately for each subplot.

As a conclusion, at some stations (AND, LYC, MAS, TRO) the directional distribution of $d \boldsymbol{H} / d t$ is strongly rotated or scattered by internal currents in the Earth's crust, but at most stations, the effect of ionospheric currents dominates.

2.6 Geomagnetically induced currents and the geoelectric field

Recordings of geomagnetically induced currents can be used to compare the diurnal occurrence of large GIC values to that of $|d \boldsymbol{H} / d t|$. In Fig. 11, the hourly distribution of GIC over $2.5 \mathrm{~A}$ at the Pirttikoski $400 \mathrm{kV}$ transformer (about $100 \mathrm{~km}$ south of SOD) is shown, as well as the corresponding distribution of GIC along the natural gas pipeline at Mäntsälä (about $40 \mathrm{~km}$ east of NUR). For more details of present GIC studies in Finland, see Pirjola et al. (1999) and Pulkkinen et al. (2000). Using the threshold of 2.5 A yields about 0.1$0.3 \%$ of all existing $10 \mathrm{~s}$ datapoints from November 1998 to December 2000. In the south, this corresponds well to the occurrence of large $d \boldsymbol{H} / d t$ at NUR (Fig. 7). At Pirttikoski, the relative number of large GIC is smaller than that of $d \boldsymbol{H} / d t$ at SOD. Using a lower limit would be a little questionable due to the accuracy of the GIC recordings. In any event, GIC at Pirttikoski shows a similar behaviour as $|d \boldsymbol{H} / d t|$ at KIL in Fig. 8. In turn, the GIC occurrence at Mäntsälä is quite similar to that of $|d \boldsymbol{H} / d t|$ at NUR. This also indicates that the sources of large $d \boldsymbol{H} / d t$ at the southern stations are partly different from those occurring in the average auroral region.

The directional distribution of the horizontal geoelectric field $\boldsymbol{E}_{h o r}$ is roughly obtained by rotating the $d \boldsymbol{H} / d t$ patterns $90^{\circ}$ anticlockwise. For example, at SOR (Fig. 9), $\boldsymbol{E}_{\text {hor }}$ would be in the east-west direction in the late evening, and then it rotates in the NS direction, which makes different times of the day different from the GIC viewpoint as well. Boteler et al. (1997) calculated the electric field from the magnetic field with the plane wave model and with the layered Earth models in Canada. Their results show that $\boldsymbol{E}_{h o r}$ tends to be in the EW direction throughout the day. However, their data selection was very different from ours: they considered 3-hour intervals in 1991 and stored only the largest $\boldsymbol{E}_{h o r}$ vector, thus obtaining 8 values per day. So they did not bin the electric field according to $|d \boldsymbol{H} / d t|$, but unavoidably included values associated with small time derivatives. Only at Ottawa, located at about the same magnetic latitude as NUR, a clear variation in directions is seen, but this is evidently due to the regular daily variation.

\section{Summary}

The original motivation for this investigation was to characterize events during which large geomagnetically induced currents occur. Then a natural selection for an activity indicator was the time derivative of the (horizontal) magnetic field, which has a close quantitative relationship to GIC. Evidently, the only previous statistical studies based directly on the time derivative are those by Coles and Boteler (1993), Boteler et al. (1997) and Viljanen (1997). A new approach in the present paper is to consider the time derivative vector, whose magnitude is a selection criterion of data. It is 


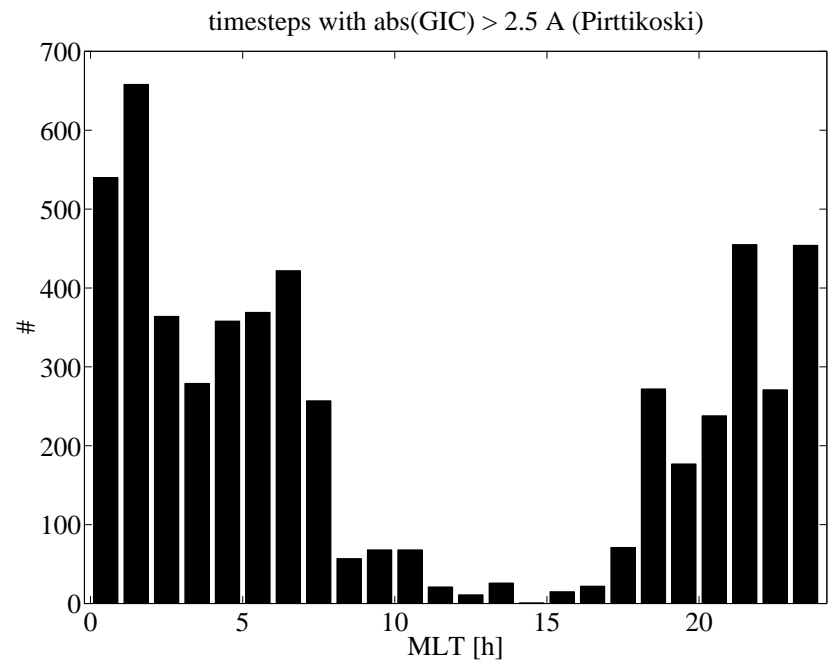

timesteps with $\operatorname{abs}(\mathrm{GIC})>2.5 \mathrm{~A}$ (Mäntsälä)

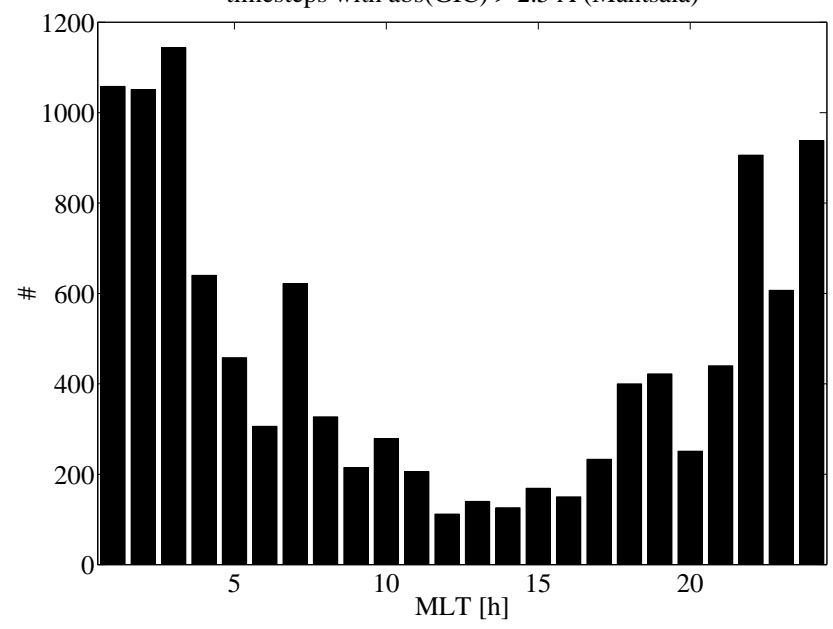

Fig. 11. Diurnal variation of the number of timesteps from 1999 to 2000 when the absolute value of GIC exceeded $2.5 \mathrm{~A}$ at the Pirttikoski $400 \mathrm{kV}$ transformer, and in the natural gas pipeline in Mäntsälä (10 s data). Compare to Fig. 8 .

not straightforward to compare our results to those by other investigators. In any event, there are several items in which our observations are in agreement with previous studies on geomagnetic activity in general, concerning time scales from solar cycles to days:

1) We found the solar cycle dependence in the sense that the probability of the occurrence of large $|d \boldsymbol{H} / d t|$ in the auroral region has its minimum around sunspot minima (Fig. 3). There is a good correlation at the yearly level with the $a a$ index;

2) Large $|d \boldsymbol{H} / d t|$ values occur more frequently in spring and autumn than in winter and summer;

3) The daily number of large $|d \boldsymbol{H} / d t|$ values (summed over all IMAGE stations) correlates well with the Nurmijärvi $A_{k}$ index. Thus, the general daily activity level can be characterized in terms of $d \boldsymbol{H} / d t$ as well;

4) Nighttime is significantly more disturbed than daytime;

5) The diurnal variation of the $d \boldsymbol{H} / d t$ activity has two max- ima: one around the local midnight and another in the morning. Except for the two southernmost IMAGE stations (NUR, UPS), this behaviour is found at all other sites during nearly all the years. Similar behaviour is known from previous ULF studies.

Some of our findings are not previously discussed in literature:

1) The diurnal distribution pattern of $d \boldsymbol{H} / d t$ varies strongly in the auroral region. The daytime rotation of the vector is clearly connected to the global ionospheric convection. The midnight substorm activity is seen as primarily north-south distributions, but a remarkable number of $d \boldsymbol{H} / d t$ vectors point to other directions as well. In the early morning, most of the large vectors are parallel to the east-west direction, which is perpendicular to the direction of the simultaneous variation field vectors;

2) The distribution pattern of $d \boldsymbol{H} / d t$ does not have any oneto-one solar cycle dependence. In particular, the distribution pattern is very narrow in the quietest and the most active years $(1987,1994)$ of our study period in the auroral region. On the other hand, it is nearly uniformly scattered during some other years. The explanation for these discrepancies may be related to different solar activity conditions, but a quantitative link to $d \boldsymbol{H} / d t$ is unknown;

3) Some seasonal variation in the distribution pattern of $d \boldsymbol{H} / d t$ in the average auroral region occurs. The pattern is narrower than particular in winter at other times; winter is geomagnetically less active than other seasons, but it remains open how this is related to the narrowness of the $d \boldsymbol{H} / d t$ pattern.

\section{Conclusions}

Our starting point was the study of large events of geomagnetically induced currents (GICs). They are characterized by large values of the time derivative of the geomagnetic field. Of special interest is the horizontal component $(d \boldsymbol{H} / d t)$, which is closely related to the (horizontal) electric field driving GIC.

A spatially and temporally extensive magnetometer database from subauroral to polar cap latitudes in Fennoscandia and Svalbard was used to derive directional distributions of the horizontal variation field vector $(\Delta \boldsymbol{H})$ and $d \boldsymbol{H} / d t$. By considering values of $|d \boldsymbol{H} / d t|$ larger than $1 \mathrm{nT} / \mathrm{s}$, it was seen that large GICs occur practically only during events characterized by westward ionospheric currents. However, distributions of $d \boldsymbol{H} / d t$ reveal that a significant amount of them are associated with structures of smaller scales than an electrojet. The distributions are systematically more scattered at auroral latitudes than in subauroral regions. Simultaneous $\Delta \boldsymbol{H}$ distributions are nearly identical at all sites and in all years. The probability of large $d \boldsymbol{H} / d t$ values increases from subauroral to auroral latitudes, but in contrast, the average value of the simultaneous $|\Delta \boldsymbol{H}|$ increases from auroral to subauroral latitudes, which underlines the peculiarity of these events. 
The directional distribution of $\Delta \boldsymbol{H}$ is easily explained by assuming a sheet current that flows parallel to the (magnetic) east-west direction. However, this model cannot explain the simultaneous $d \boldsymbol{H} / d t$ distributions. The apparent conflict is resolved by stating that, in addition to the main electrojet, there are localized structures in which the current can flow in any direction. Current amplitudes in such structures can be small, and therefore do not affect the $\Delta \boldsymbol{H}$ distribution. The point is that the associated temporal changes are so fast that they are seen in the $d \boldsymbol{H} / d t$ distribution. In magnetograms, this is observed as a large $\Delta X$ (compared to $\Delta Y$ ), but about equal $d X / d t$ and $d Y / d t$ exists during events with a large $|d \boldsymbol{H} / d t|$. (In other words, a small $\Delta Y$ variation does not imply a small $d Y / d t$.)

Data from a subset of five stations located in the average auroral region indicate clear differences in the $d \boldsymbol{H} / d t$ distributions from 1982 to 2000 . For example, there are two years (1987 and 1994) with a narrow directional distribution at all five stations, with 1987 being a very quiet year in the $|d \boldsymbol{H} / d t|$ sense, whereas 1994 was the most active year in the study period. On the other hand, 1998, with an average activity (and exactly one sunspot cycle later than 1987), has nearly a uniformly scattered $d \boldsymbol{H} / d t$.

Our results imply that the geoelectric field, which is closely related to $d \boldsymbol{H} / d t$, can be large in any direction, not only parallel to the main electrojet. Consequently, when statistical predictions of GIC are derived from geomagnetic data, it is necessary to include a representative sample of events of several years. Additionally, it should be remembered that although large GIC primarily occur during westward electrojets, there are major events of a completely different kind, such as sudden impulses, which as rare phenomena are hidden in the statistics.

Our starting point was quite different from some previous studies which covered several years and used data of global indices (AL, AU, AE), or extensive time series of auroral observations. Results are not directly comparable, but in any event, those investigations have also revealed statistical changes from one solar cycle to another. An especially interesting finding is the strong concentration of large $d \boldsymbol{H} / d t$ vectors to the east-west direction in the morning, transverse to the simultaneous main flow of ionospheric currents to the west. This may be related to the pulsation activity, and would require an investigation of small period variations binned according to the $|d \boldsymbol{H} / d t|$ level.

Returning to the original motivation of this study (GICs), an important future work is to perform case studies of large $|d \boldsymbol{H} / d t|$ events to investigate their morphology. As the final aim, quantitative descriptions of ionospheric currents are needed, i.e. the current density as a function of time and space. After that, it is straightforward to determine GICs (Viljanen et al., 1999). There is presently ongoing work to derive a complete two-dimensional description of ionospheric equivalent currents above Fennoscandia, based on the method by Amm and Viljanen (1999). A special task is to study large events occurring at subauroral latitudes, where most of the systems prone to GIC are located in Finland and elsewhere in the world. It is interesting to find out whether extreme GIC events are just due to auroral currents shifted equatorward, or whether they are qualitatively different, as our study indicates.

Acknowledgement. IMAGE magnetometer data are collected as an international project headed by the Finnish Meteorological Institute (http:www.geo.fmi.fi/image/). The EISCAT magnetometer cross was operated as a German-Finnish collaboration lead by the Technical University of Braunschweig. Data of geomagnetically induced currents in the Finnish high-voltage power system are provided by Fingrid Oyj, and the current in the Finnish natural gas pipeline is recorded in co-operation with Gasum Oy. The work of Antti Pulkkinen was supported by the Academy of Finland. We are also acknowledged to Johanna Juntunen and Eija Tanskanen for their help in determining quiet time baselines for IMAGE data. Pekka Janhunen helped with the calculation of magnetic local times. Kirsti Kauristie and Olaf Amm, as well as the two referees, are acknowledged for very useful comments on the manuscript. Special thanks go to numerous persons who have been involved in the data verification.

Topical Editor M. Lester thanks S. Vennerstrøm and another Referee for their help in evaluating this paper.

\section{References}

Ahn, B.-H., Kroehl, H. W., Kamide, Y., and Kihn, E. A., Seasonal and solar cycle variations of the auroral electrojet indices, J. Atmos. Sol.-Terr. Phys., 62, 1301-1310, 2000.

Albertson, V. D., Kappenman, J. G., Mohan, N., and Skarbakka, G. A., Load-flow studies in the presence of geomagneticallyinduced currents, IEEE Trans. Power Appar. Syst., PAS-100, 594-607, 1981.

Amm, O., Direct determination of the local ionospheric Hall conductance distribution from two-dimensional electric and magnetic field data: Application of the method using models of typical ionospheric electrodynamic situations, J. Geophys. Res., 100, 21473-21488, 1995.

Amm, O. and Viljanen, A., Ionospheric disturbance magnetic field continuation from the ground to the ionosphere using spherical elementary current systems, Earth, Planets and Space, 51, 431440, 1999.

Bolduc, L., Langlois, P., Boteler, D., and Pirjola, R., A study of geoelectromagnetic disturbances in Québec, 1. General results, IEEE Trans. Power Delivery, 13, 1251-1256, 1998.

Boteler, D. H., Boutilier, S., Bui-Van, Q., Hajagos, L., Swatek, D., Leonard, R., Hughes, B., Ferguson, I. J., and Odwar, H. D., Geomagnetic Hazard Assessment, Phase 2, Final Report, CEA project 357 T 848A, GSC Open File 3140, 1997.

Boteler, D. H., Pirjola, R. J., and Nevanlinna, H., The effects of geomagnetic disturbances on electrical systems at the Earth's surface, Adv. Space Res., 22, 17-27, 1998.

Coles, R. L. and Boteler, D. H., Geomagnetic induced currents: assessment of geomagnetic hazard, GSC Open File 2635, 1993.

Coles, R. L., Thompson, K., and Jansen van Beek, G., A comparison between the rate of change of the geomagnetic field and geomagnetically induced currents in a power transmission system, Proc. EPRI Conf. Geomagnetically Induced Currents, Burlingame, Ca., 8-10 Nov. 1989, EPRI TR-100450, 15, 1-8, 1992. 
Hajkowicz, L. A., Longitudinal (UT) effect in the onset of auroral disturbances over two solar cycles as deduced from the AEindex, Ann. Geophys., 16, 1573-1579, 1998.

Korja, T. and Hjelt, S.-E., Electromagnetic studies in the Fennoscandian Shield - electrical conductivity of Precambrian crust, Phys. Earth Planet. Inter., 81, 107-138, 1993.

Lühr, H., Thürey, S., and Klöcker, N., The EISCAT-Magnetometer Cross. Operational Aspects - First Results, Geophys. Surv., 6, 305-315, 1984.

Mäkinen, T., Geomagnetically induced currents in the Finnish power transmission system, Finn. Meteorol. Inst. Geophys. Publ., 32, 101 pp., 1993.

Nevanlinna, H. and Pulkkinen, T.I., Auroral observations in Finland: Results from all-sky cameras, 1973-1997, J. Geophys. Res., 106, 8109-8118, 2001.

Olson, J. V., ULF Signatures of the Polar Cusp, J. Geophys. Res., 91, 10055-10062, 1986.

Pajunpää, K., Conductivity anomalies in the Baltic Shield in Finland, Geophys. J. Roy. Astr. Soc., 91, 657-666, 1987.

Pirjola, R. and Viljanen, A., Complex image method for calculating electric and magnetic fields produced by an auroral electrojet of finite length, Ann. Geophysicae, 16, 1434-1444, 1998.

Pirjola, R., Pulkkinen, A., Viljanen, A., Nevanlinna, H., and Pajunpää, K., Study Explores Space Weather Risk to Natural Gas Pipeline in Finland, EOS Transactions, 80, 332-333, 1999.

Pulkkinen, A., Viljanen, A., Pirjola, R., and BEAR Working Group, Large geomagnetically induced currents in the Finnish highvoltage power system, Finn. Meteorol. Inst. Rep., 2000: 2, 99, 2000.

Rasmussen, T. M., Roberts, R. G., and Pedersen, L. B., Magnetotellurics along the Fennoscandian Long Range Profile, Geophys. J. R. astr. Soc., 89, 799-820, 1987.

Russell, C. T. and McPherron, R. L., Semiannual Variation of Geomagnetic Activity, J. Geophys. Res., 78, 92-108, 1973.

Syrjäsuo, M. T., Pulkkinen, T. I., Janhunen, P., Viljanen, A., Pelli- nen, R. J., Kauristie, K., Opgenoorth, H. J., Wallman, S., Eglitis, P., Karlsson, P., Amm, O., Nielsen, E., and Thomas, C., Observations of substorm electrodynamics using the MIRACLE network, in: Proceedings of the International Conference on Substorms-4, Editors: S. Kokubun and Y. Kamide, Astrophysics and Space Science Library, vol. 238, Terra Scientific Publishing Company/Kluwer Academic Publishers, 111-114, 1998.

Towle, J. N., Prabhakara, F. S., and Ponder, J. Z., Geomagnetic effects modelling for the PJM interconnection system. Part I Earth surface potential computation, IEEE Trans. Power Syst., 7, 949-955, 1992.

Untiedt, J. and Baumjohann, W., Studies of polar current systems using the IMS Scandinavian magnetometer array, Space Science Reviews, 63, 245-390, 1993.

Vennerstrøm, S., Dayside magnetic ULF power at high latitudes: A possible long-term proxy for the solar wind velocity?, J. Geophys. Res., 104, $10145-10157,1999$.

Viljanen, A. and Pirjola, R., Geomagnetically induced currents in the Finnish high-voltage power system - a geophysical review, Surv. Geophys., 15, 383-408, 1994.

Viljanen, A., The relation between geomagnetic variations and their time derivatives and implications for estimation of induction risks, Geophys. Res. Lett., 24, 631-634, 1997.

Viljanen, A., Relation of Geomagnetically Induced Currents and Local Geomagnetic Variations, IEEE Trans. Power Delivery, 13, 1285-1290, 1998.

Viljanen, A., Kauristie, K., and Pajunpää, K., On induction effects at EISCAT and IMAGE magnetometer stations. Geophys. J. Int., 121, 893-906, 1995.

Viljanen, A., Amm, O., and Pirjola, R., Modelling Geomagnetically Induced Currents During Different Ionospheric Situations, J. Geophys. Res., 104, 28 059-28 072, 1999.

Ziesolleck, C. W. S. and McDiarmid, D. R., Statistical survey of auroral latitude Pc 5 spectral and polarization characteristics, J. Geophys. Res., 100, 19299-19312, 1995. 